\title{
El Periodismo Cultural
}

DOI: http://dx.doi.org/10.5377/koot.v0i9.5906

URI: http://hdl.handle.net/11298/436

\author{
Néstor Martínez \\ Periodista / Escritor \\ nestormarven@gmail.com
}

\section{Resumen}

Cultura y Periodismo, una mezcla que involucra un campo demasiado amplio y otro demasiado restringido. Sin embargo, tiene roces comunes en los que interviene el Periodista, armonizando esos conceptos para el público en general. El presente artículo nos acerca a la teorización de esa armonización, al tiempo que nos revela algo de la faceta del Periodismo Cultural.

Palabras clave: Periodismo, Periodismo histórico, Cultura, Difusión de la cultura.

\begin{abstract}
Culture and journalism, a mixture that involves both a very broad field and very restricted one. However, hey have common frictions in which the journalist mediates by harmonizing these concepts for the general public. This article brings a closer look of the theorizing of this harmonization, revealing simultaneously aspects of Cultural Journalism, for the country.

Keywords: Journalism, Historical journalism, Culture, Cultural Diffusion.

\section{Introducción}

Periodismo y cultura, son dos conceptos muy difíciles de unir. Por un lado tenemos que el concepto de Cultura es más amplio que el de Periodismo, y por el otro tenemos que el reducido concepto de Periodismo, lo convierte en una actividad limitada a la simple acción y reacción reporteril de informar para el público. El suceso, la acción, provoca la reacción del periodista, que echa mano de sus herramientas profesionales para trasladar los elementos noticiosos que considera del interés para el gran público. Así que, para encontrar una aproximación práctica que nos abra el camino hacia la definición y práxis del Periodismo Cultural, habrá que reducir el concepto de Cultura hasta adaptarlo al Periodismo.
\end{abstract}


María J.Villa, en la investigación sobre Periodismo Cultural, para su tesis doctoral, ${ }^{1}$ plantea el problema de esta manera:

"Partimos de la idea de que el periodismo cultural se aplica a un campo extenso y heterogéneo. Esta primera aproximación nos marca la imposibilidad de ser abordado desde una sola perspectiva. Involucra y excluye a los géneros y productos del campo periodístico produciéndose una constante pendulación entre los términos "periodismo" y "cultura". La problemática inicial estaría centrada en ver cuál es el concepto de cultura que está detrás del llamado periodismo cultural.
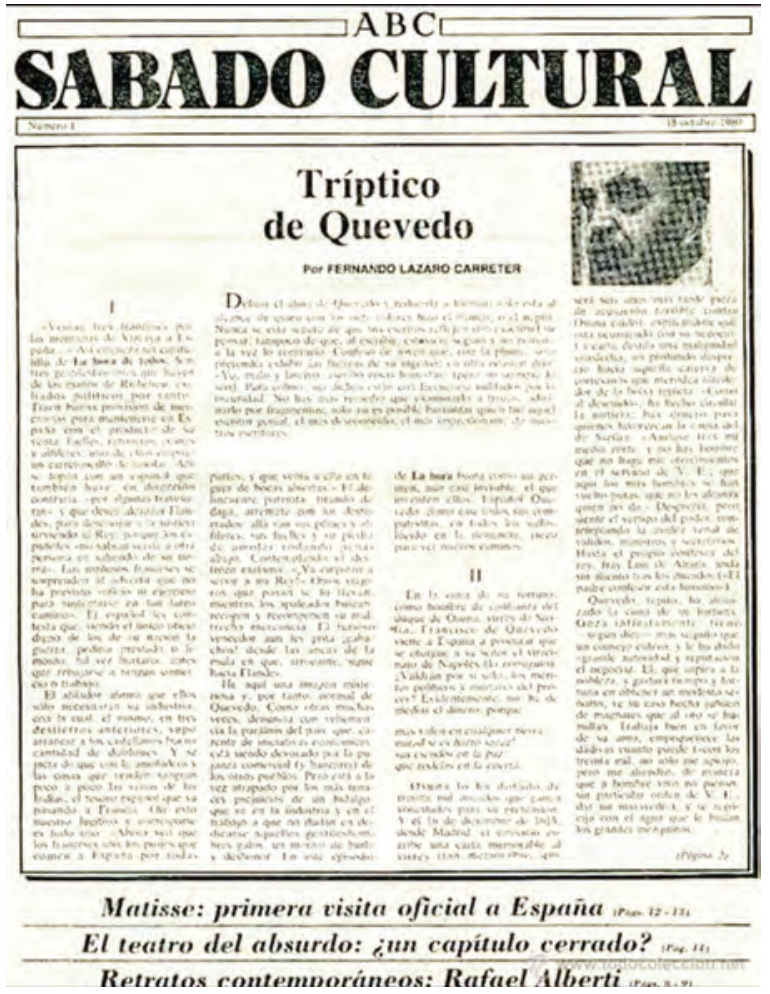

Sabemos que el término "cultura" fue abordado desde diferentes disciplinas y posturas ideológicas. Sin embargo, hay dos concepciones básicas a tener en cuenta en un primer momento. Una concepción, que proviene de la ilustración y que generalmente es aplicada a un grupo de personas que detentan el "saber" y el "buen gusto", esta posición califica positiva o negativamente, divide los grupos en "cultos" o "incultos", despreciando la capacidad de todos los sujetos para hacer cultura. Otra, más amplia e integradora se sostiene por los aportes de la antropología cultural”.

1 Revista LATINA de Comunicación Social La Laguna (Tenerife) - número 6 - junio de 1998. 


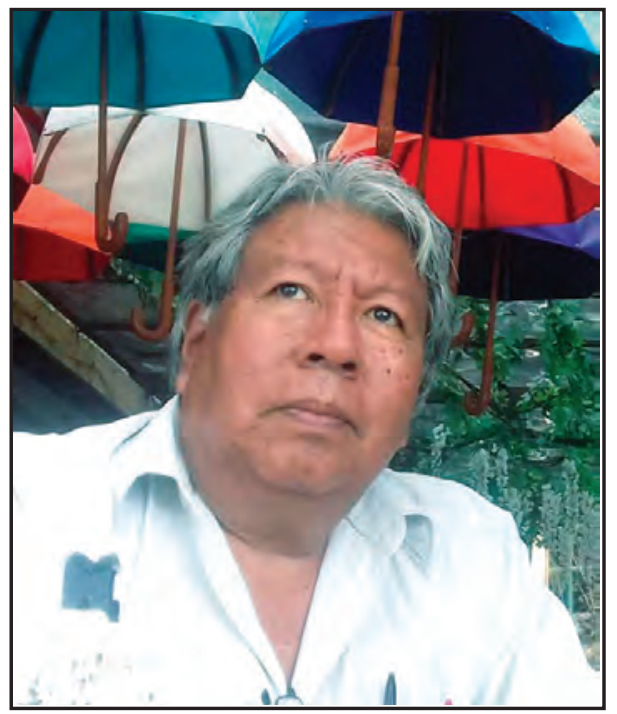

Licenciado y Periodista Néstor Martínez.

El sociólogo mexicano Jorge González, quien, preocupado por reflexionar sobre las problemáticas culturales en América Latina, define a la cultura como: "Un modo de organizar el movimiento constante de la vida concreta, mundana y cotidianamente. La cultura es el principio organizador de la experiencia, mediante ella ordenamos y estructuramos nuestro presente, a partir del sitio que ocupamos en las redes de las relaciones sociales"2

Desde estos dos puntos de vista, habría material suficiente para empezar a elaborar el marco en que el Periodismo Cultural se ejerce o debería de ejercerse. Pero agregaré un concepto que amplía el debate.

En su estudio "Los dos conceptos de cultura, entre la oposición y la confusión"3, Irene Martínez Sahuquillo, sostiene que "debido al efecto mistificador de corrientes como el multiculturalismo y de ciertas ciencias sociales, la cultura, en el sentido restringido, está siendo erosionada y deslegitimada, a la vez que se tienden a borrar las fronteras que dividen a la alta cultura de las masas, así como de la cultura en el sentido antropológico".

\section{Un concepto conciliador es el de la UNESCO:}

Cultura es la que da al hombre la capacidad de reflexionar sobre sí mismo. Es ella la que hace de nosotros seres específicamente humanos, racionales, críticos y éticamente comprometidos. A través de ella discernimos los valores y efectuamos opciones. A través de ella el hombre se expresa, toma conciencia

2 Idem.

3 Los dos conceptos de cultura: Entre la oposición y la confusión. Martínez Sahuquillo, Irene (REIS No 79. ESTUDIOS). Revista Española de Investigaciones Sociológicas 1978-2002. 
de sí mismo, se reconoce como un proyecto inacabado, pone en cuestión sus propias realizaciones, busca incansablemente nuevas significaciones, y crea obras que lo trascienden.

A estos cuatro escenarios: cultura elitista, cultura como todo el modo de vida humana, y la confusión que provocan los diferentes conceptos de cultura, le agregaremos el escenario limitado de Periodismo.
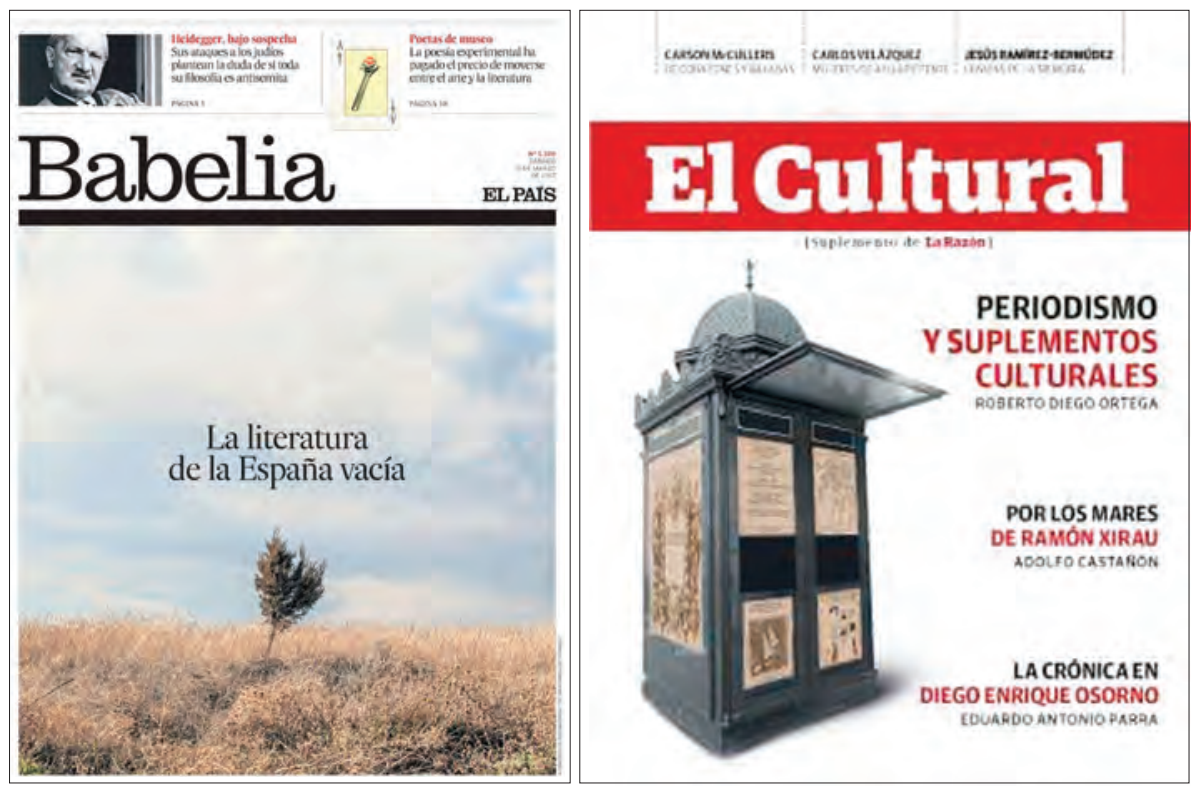

No es muy complicado entender lo que es el Periodismo

- Actividad cuyo fin es recolectar, sintetizar, jerarquizar y publicar información relativa a hechos del presente, del pasado y/o del futuro. En este sentido, el periodismo se entiende como una metodología adecuada para poder presentar cualquier tipo de información valiosa, buscar fuentes seguras y verificables.

- Profesión que comprende el conjunto de actividades relacionadas con la obtención, elaboración y difusión de información actual o de interés para transmitirla al público a través de los medios de comunicación.

- Conjunto de estudios o conocimientos necesarios para obtener un título académico de periodista. Dada la evidente influencia del periodismo en la sociedad, se ha desarrollado una deontología profesional constituida por una serie de normas y deberes éticos -ética periodística-, que guían la actividad del periodista. Dichos códigos deontológicos son emitidos generalmente por los colegios profesionales en los países en que estos existen. En general, 
estos códigos postulan la independencia de los medios respecto a los poderes políticos y económicos. El periodista queda sujeto a su obligación de actuar con la mayor diligencia posible en el acceso a las fuentes y en el contraste de opiniones confrontadas.

\section{Estos conceptos se encuentran en cualquier estudio sobre el Periodismo}

La demanda de la sociedad de ser informada en diversos ámbitos, o la necesidad de difundir diversas actividades humanas del interés de la sociedad, nos lleva a la división del Periodismo, según sea la especialización a que el profesional quiera aplicar sus conocimientos. De esta manera tenemos, Periodismo Científico, Periodismo Deportivo, Periodismo Cultural, Periodismo Ambiental, etcétera. Cada especialidad conlleva el conocimiento propio del ámbito elegido.

Iván Tubau, en su libro Teoría y práctica del periodismo cultural (1982)[4]define al periodismo cultural como "la forma de conocer y difundir los productos culturales de una sociedad a través de los medios de comunicación masivos".

Otro concepto difundido por Internet: Es la rama del oficio periodístico dirigida a cubrir todas las manifestaciones del amplio concepto que abarca el término de cultura en el día a día de una sociedad. ${ }^{4}$

Ambos conceptos, con la pretensión de ser precisar lo que es el Periodismo Cultural, nos dejan en el limbo de lo qué son "productos culturales" o qué hay detrás de "el término cultura".

En el concepto de Tubau, podemos caer en la no tentadora trampa que nos plantea María J.Villa: la del periodismo elitista, discriminador, mientras que en el segundo concepto nos mete en una camisa demasiado grande para el Periodismo Cultural, tal como González define cultura, como la degeneración del concepto cultura que plantea Martínez Sahuquillo, o como preguntarse cuál es el concepto de cultura que debo manejar.

Pese a este aparente confuso mar, aún es posible navegar con el auxilio del salvavidas conceptual: El Periodista especializado debe traducir los códigos de su especialización para que todo tipo de público tenga acceso al conocimiento, digamos, propio de una elite científica o cultural, reduciendo en este caso la cultura al ámbito de las bellas artes. En este concepto adquiere dimensión la función educadora del periodista que trasciende la simple función informadora, más allá aún: en los hombros del Periodista Cultural, recae el que las artes, como la parte más sublime del espíritu humano, sean despojadas de la investidura solo para cultos y ponerle el ropaje del pueblo llano.

El Periodista Cultural debe darle vida y praxis al concepto de la cultura de la UNESCO, que amplía el panorama cultural: no solo existen las bellas artes como

4 Teoría y práctica del Periodismo Cultural, Iván Tubau, A.T.E. Argentina. 1982. 
propias de una elite que las manifiesta, sino que hay expresiones culturales entre el pueblo o las comunidades indígenas de un país, que a través de la difusión de sus propias costumbres, ritos, y expresiones culturales de todo tipo, mantienen la identidad, la cohesión de la sociedad, su historicidad que es transmitida de generación a generación.

Cultura viene del latín Cultus que significa cultivo. Esa es la misión del Periodismo Cultural: que el ser humano cultive su más alta manifestación espiritual: las artes, cualquiera que el ámbito en que se manifiesten, o las manifestaciones culturales que definen e identifican a los diversos pueblos.

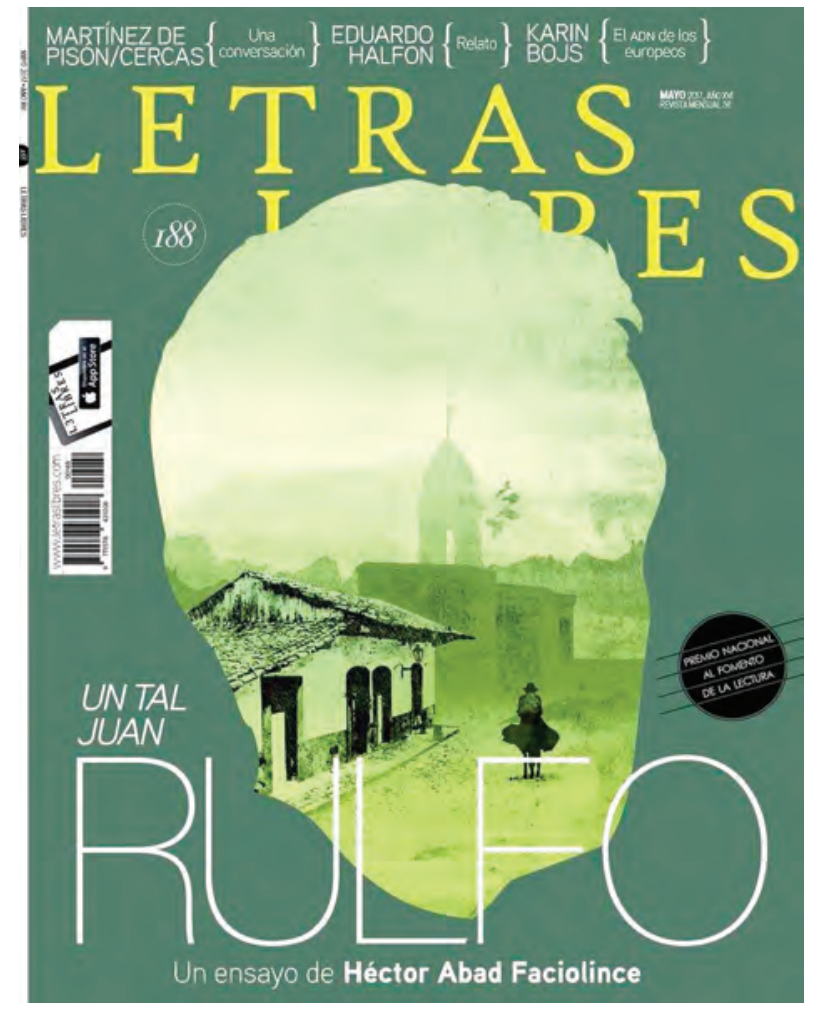

\section{Referentes Bibliográficos}

Fundación Wikimedia, Inc. (1982). Teoría y práctica del Periodismo Cultural. Recuperado de https://es.wikipedia.org/wiki/Periodismo_cultural

Martínez Sahuquillo, I. (1997). Los dos conceptos de cultura: entre la oposición y la confusión. Revista Española de Investigaciones Sociológicas. (79) 173-196.

Villa, M. J. (1998). El periodismo cultural: reflexiones y aproximaciones. Revista Latina de Comunicación Social La Laguna. Recuperado de https://www.ull.es/publicacione s/latina/a/83mjv.htm. 